\title{
Instructional Design in the Formation of Mental Images and the Genetic Decomposition of a Concept
}

\author{
Dr Zingiswa Mybert Monica Jojo
}

jojozmm@unisa.ac.za

\section{Doi:10.5901/jesr.2014.v4n3p139}

\begin{abstract}
This paper reports on the instructional design used in an APOS (Actions, Processes, Objects and Schema) approach to difficulties experienced by first year engineering students at a University of Technology in constructing the concept of the chain rule. Instructional treatment followed the activities, classroom discussions and exercises (ACE) model as specified by a proposed genetic decomposition of the chain rule concept in differential calculus. This paper in particular presents instructional design used on three sequential lessons that were video recorded during class time in slots of one hour duration whilst introducing the chain rule to a class of about 197 first year students. Those lessons followed immediately after the students had done an introduction to calculus, differentiation using the product and quotient rules. Here I discuss in brief the techniques used in those lessons, instructional strategy used and the interactions between the researcher and the students, students to student collaborations in the classrooms. This was a qualitative study where the results revealed to a greater extent the effect of instructional design to an object understanding of the chain rule concept. The students' interactions indicated that the instructional pedagogy should accommodate presentation of tasks that evoke rigorous deductive reasoning enabling the students to write and reflect on how they construct various mental images. A wide range of interactions between students themselves and between students and the researcher revealed that most students preferred the straight form technique and barely ever used Leibniz technique when differentiating loaded trigonometric functions.
\end{abstract}

Keywords: APOS, Instructional design, trigonometric functions, chain rule, interactions, straight form technique, link form technique, Leibniz form technique

\section{Introduction}

The chain rule is the underlying concept in many applications of calculus: implicit differentiation, solving related rate of change problems, applying it in the fundamental theorem of calculus and solving differential equations. APOS has been very useful in attempting to understand students' learning of a broad range of topics in calculus, abstract algebra, statistics, discrete mathematics, and other areas of undergraduate mathematics (Dubinsky et al, 1991), and to suggest ways that students can learn these concepts (Dubinsky and Mc Donald (2001). The latter further suggest that this theory can also point us towards pedagogical strategies that lead to marked improvement in (1) student learning of complex or abstract mathematical concepts, and (2) students' use of these concepts to prove theorems, provide examples, and solve problems.

In extension of reflective abstraction theory, Dubinsky (1991) isolated some essential features of reflective abstractions, reorganized and reconstructed them to form a coherent theory of mathematical knowledge and its construction, APOS (Actions, Process, Object, and Schema) whose concepts are elaborated further on, in Maharaj (2010:43). A flow diagram to illustrate APOS extended in explaining the activities involved in construction of the chain rule concept can be found in Jojo (2011:61). This flow diagram illustrates diagrammatically the coordination of processes involved in reflective abstraction, APOS and the Triad mechanism.

The chain rule states that if $g(x)$ is a function differentiable at $c$ and $f$ is a function differentiable at $g(c)$, then, the composite function $f o g$ given by $(f o g)(x)=f(g(x))$ is differentiable at $c$ and that $(f o g)^{\prime}(c)=f^{\prime}(g(c)) \cdot g^{\prime}(c)$. Calculus is one of the topics introduced to matric learners at high school, yet a large number of students receive inadequate mathematics education and join the university mostly under-prepared for the study of differential calculus. Informal discussions held with other lecturers, revealed that the chain rule is one of the most complicated calculus tools, despite being one of the basic tools for a mathematician. Furthermore the chain rule is now part of the matric South African school syllabus. In my experience many first year university students have difficulty in understanding the chain rule in differentiation.

The chain rule is of important use to other areas of calculus. These include: (1) Finding the marginal Physical 
Productivity Function of the workers in Business economics $\left(\frac{d P}{d x}\right)$ for $P=10(3 x+2)^{3}-10$, (2) Revenue changing when given a revenue function like $R(x)=25(x+2)^{2}+20 x-5$, (3) Higher order differentiation used to calculate demand, cost and profit in business and (4) Calculations of rates on physical body relationships including body weight and surface area, cell growth, blood flow and other physical quantities. It is important for the students at this stage to know which formula to use and how to use it without computing the derivatives of the component functions. They must be able to identify whether a constant times a function, sum of functions, product, quotient, composition or piecewise functions are given in the problem. The implication here is that they should be well versed with function algebra.

The chain rule is included in several studies in mathematics education literature. Some of them are about teaching of the chain rule (Lutzer, 2003; Mathews, 1989; Thoo 1995; Uygur \& Ozdas, 2007) while others are on understanding the rule. Uygur (2010) who studied the cognitive development of applying the chain rule through the three worlds of mathematics suggested that the instructional way of presenting the chain rule changed focus to encourage students to obtain the chain rule with some life-related problem situations. In contrast, verifying the chain rule by using either or both graphing software or graphics calculator and an algebraic approach was considered for developing teaching and learning strategies of the chain rule in the mathematics teaching program of South Australia (SACE Board of South Australia, 2009). Uygur (2010) further noted that as much as there was an absence of studies on structural development of the chain rule, there was also a need for a study on students' applying the chain rule to second order derivatives and to twovariable composite functions. It was noted also, that the prerequisite knowledge of composite function is another significant notion for applying the chain rule by raising awareness of the relation among various cases. Uygur inferred that variable notion is another significant prerequisite knowledge in the embodied world of the cognitive development of the chain rule.

Novotna and Hoch (2008) had indicated the importance of structural knowledge in applying the chain rule in the cognitive development of mathematical concepts. Students' application of the chain rule was analysed within Tall's (2007) framework containing three levels of understanding which considered symbolic development. Their study addressed the structural development of the chain rule. On the contrary this study focused on the discussion of the types of structures constructed by students when learning the chain rule with the view to clarifying their understanding: (i) of the composition of function and (ii) of the derivative.

Cottrill (1999) conducted a study on the understanding of the chain rule asserted that: (1) conventional wisdom holds that students' conception of the chain rule (as with other rules) is that of symbol manipulation, (2) the conception of the chain rule appeared to be a straight-forward manipulation of symbols which could easily be applied in problem situations and (3) concluded that an application based on symbol manipulation carries a heavy requirement for the function to be given by an expression, fostering students' tendencies toward instrumental understanding, where they are unable to apply the chain rule.

Conceptual knowledge as defined by Hapasaalo (2004) denotes knowledge of and a skilful "drive" along particular networks, the elements of which can be concepts, rules (algorithms, procedures, etc.), and even problems (a solved problem may introduce a new concept or rule) given in various representation forms. Various researchers (Confrey, 1994; Gagnon \& Collay, 2001; Piaget, 1948/1973; Pirie \& Kieren, 1994) assert that the degree of understanding is determined by the number and the strength of the connections made with previously acquired mathematics. Thus a mathematical idea, procedure, or fact is understood thoroughly if it is linked to existing networks with stronger or more numerous connections. It is my view therefore that instructional design for this concept should incorporate strategies that bring students to relational understanding of the concept such that they can be able to use it in life-related applications and in greater calculus.

\section{Literature Reviewed}

Hiebert \& Carpenter (1992) asserted that learning mathematics with understanding involves making connections among ideas, and that those connections are considered to facilitate the transfer of prior-knowledge to novel situations. With regard to the psychological approach to learning, the constructivist idea is that understanding is a continuing activity of individuals organizing their own knowledge structures, a dynamic process rather than an acquisition of categories of knowing (Confrey, 1994; Gagnon \& Collay, 2001; Piaget, 1966; Pirie \& Kieren, 1994). According to Bransford, Brown \& Cocking, (2000), a mathematical idea or procedure or fact is understood if it is part of an internal network. More specifically, the mathematics is understood if its mental representation is part of a network of representations. They further asserted that the degree of understanding is determined by the number and the strength of the connections made 
with previously acquired mathematics. Thus a mathematical idea, procedure, or fact is understood thoroughly if it is linked to existing networks with stronger or more numerous connections. It is therefore assumed that well-connected and conceptually grounded ideas enable their holders to both remember them and see them as part of a larger whole within which each part shares reciprocal relationships with other parts, (Pirie \& Kieren, 1994).

Wiggins, (1993) defined understanding as something different that emerges when we are required to reflect upon achievement, in verifying or criticizing, re-thinking and re-learning what we know. Wiggins \& McTighe (1998) further identified several inter-related aspects of understanding including (1) explanation, (2) interpretation, (3) contextual applications, (4) perspective, (5) empathy and (6) self-knowledge. Not all of these apply to each learning situation and they are not hierarchical or mutually exclusive. Students who can explain their ideas justify understanding by making connections and inferences. Those who apply knowledge demonstrate their ability to use what they have learnt in complex situations. Lastly, those who show self-knowledge recognize the limits of their understanding.

Dubinsky (1991 a) believed that reflective abstraction as introduced by Piaget (Berth \& Piaget, 1966) is a powerful tool in the study of advanced mathematical thinking and that it could be used to analyse any mathematical knowledge applicable to higher education. Reflective abstraction is a concept introduced by Piaget to describe the construction of logico-mathematical structures by an individual during the course of cognitive development. He made two important observations: first, reflective abstraction has no absolute beginning, but is present at the earliest ages in the coordination of the sensori-motor structures (Beth \& Piaget, 1966) and second, that it continues on up through higher mathematics to the extent that the entire history of the development of mathematics from antiquity to the present day may be considered as an example of the process of reflective abstraction.

Piaget distinguished three major kinds of abstraction which are not independent of each other. He talks of empirical knowledge which derives knowledge from properties of objects, (Beth \& Piaget, 1966). According to Piaget, (Piaget \& Garcia, 1983), this kind of abstraction leads to extraction of common properties of objects and extensional generalizations that is, the passage from specific to general. Pseudo-empirical is intermediate between empirical and reflective abstraction and it spells out properties that the actions of the subject have introduced into objects, (Piaget, 1985). Finally, reflective abstraction is drawn from what Piaget (1980) called general co-ordinations of actions by the subject internally. He asserts that this kind of abstraction leads to a very different sort of generalization which is constructive and results in new syntheses in the midst of which particular laws acquire new meaning (Piaget \& Garcia,1983).

There were many examples of instances of reflective abstractions, but I can site that when a student was given a problem like, 'Differentiate: $y=\left(\operatorname{cosec}^{3} x+e^{\tan x}\right)^{2}$,' he performed several individual actions in his mind to identify the different functions involved. He would then interiorise and coordinate the actions to form a total ordering of where to start differentiating. Those actions would form new actions, and ultimately new objects (which may no longer be physical but rather mathematical such as a new function derived from the original one). Piaget (1972) further asserted that it is reflective abstractions in its most advanced form that leads to the kind of mathematical thinking by which form or process is separated from the content and that processes themselves are converted, in the mind of the mathematician, to objects of content, (Piaget, 1972). Empirical abstraction therefore deals with action as opposed to objects and it differs from pseudo-empirical abstraction in that it is concerned, not so much with the actions themselves, but with interrelationships among actions, which Piaget (1975) called 'general co-ordinations.'

According to Piaget, the first part of reflective abstractions consists of drawing properties from mental or physical actions at a particular level of thought (Beth \& Piaget, 1966). He says that this involves consciousness of the actions, and can include the act of separating a form from its content. Whatever is abstracted is projected onto a higher plane of thought where other actions are present as well as more powerful modes of thought. For further elaboration on the five kinds of construction in reflective abstraction namely: Interiorisation; coordination, encapsulation, generalization and reversal, see Jojo, Brijlall and Maharaj (2013).

Weyer (2010) asserts that the first circumstance to happen should be something that happens as a result of instructional practice. Also constructivists, Piaget (1966) and Vygotsky (1986) have insisted that the mental constructions that a person uses to understand a mathematical concept are made in a social context and with considerable intervention from teachers and fellow students. Thus mathematics pedagogy based on the said theories approaches mathematics as a conceptual system rather than discrete procedures. Genuine education depends both on the knowledge and experience already existing within a student's level of development as well as on the student's potential to learn (Vygotsky, 1986). Beth and Piaget, (1966) argue that if we observe a person moving from 'not understanding a concept' to 'understanding it', we cannot be able to see what mental mechanisms are used, also we cannot tell if these mechanisms are used to construct the concept or to gain access to it. Thus the researcher felt it necessary to video- 
record the students in action so that we could go beyond observation and consider their conversations, written texts and their interactions with peers and instructor.

Interventions and selected approaches were used to strengthen the learning aspirations of the students. As an experienced lecturer I had to use scaffolding to help the learners to move from one level of mathematical understanding to another. Tools and strategies which assist students to attain a higher level of understanding by encouraging creative and divergent thinking are known as scaffolds (Brush \& Saye, 2001; Mccosker \& Diezman, 2009). Anghileri (2006) asserts that students actively construct meaning as they engage significantly within established mathematical practices. These tools in a mathematics classroom could include diagrams, pictures, technology, mathematics formulas and hints for an effective solution process.

Ernest (1991) asserts that Mathematics Education understood in its simplest and most concrete sense concerns the activity or practice of teaching mathematics. He further asserts that learning is inseparable from teaching. This process involves the exercise of the mind and intellect in thought, enquiry, and reasoning. Similarly, the interpretive research paradigm used in this study explored the real human and social situations and uncovered the meanings, understandings and interpretations of the participants involved. It was therefore evident that in exploring how students conceptualized the understanding of the chain rule, APOS could be used objectively to (1) explain students' difficulties with the chain rule and (2) suggest ways that students can learn the chain rule. More specifically APOS could lead us towards pedagogical strategies that in turn lead to marked improvement in (1) student learning of the chain rule and (2) students' use of this concept to solve problems in calculus.

\section{Framework}

APOS ascertains that to understand a mathematical concept, one must begin with manipulating previously constructed mental or physical objects in the learner's mind to form actions; actions would then be interiorised to form processes which are then encapsulated to form objects. These objects could be de-encapsulated back to the processes from which they are formed, which would be finally organized in schemas. This explained the theoretical analysis which covered the first part of the APOS framework. The other parts of the framework are the instructional design used and data collection methods. All three are explained in detail in Jojo (2013). This paper reports on the instructional activities followed during the teaching of three sequential lessons to establish the understanding of the concept of the chain rule.

Understanding of the chain rule in this study was conceptualised in relation to the schema relevant to it. A schema for a certain mathematical concept is an individual's collection of actions, processes, objects and other schemas which are linked by some general principles to form a framework in the individual's mind that may be brought to bear upon a problem situation involving that concept (Dubinsky \& Mc Donald, 2000). Asiala et al, (2004) asserted that an individual's schema is the totality of knowledge which for her is connected consciously or unconsciously to a particular mathematical topic, for example an individual may have a function schema, derivative schema, a chain rule schema. The chain rule schema includes a student's version of the concept described by the genetic decomposition, as well as other concepts that are perceived to be linked to the chain rule. A structured set of mental constructs which might describe how the concept can develop in the mind of an individual is called the genetic decomposition of that particular concept, Dubinsky (1991).

The genetic decomposition of the concept of the chain rule suggested below was used to guide the researcher's teaching instruction in class. It was believed that the chain rule schema develops through the levels of the triad: Intra, Inter and Trans. (i) In the first level, the Intra- level, the student shows evidence of having a collection of rules for finding derivatives of functions in various situations, but has no recognition of the relationships between them. This collection may include some special cases of the chain rule, and perhaps even the general formula which is perceived as a separate rule rather than a generalization of the others. (ii) The Inter-level is characterized by the student's ability to begin to (mentally) collect all elements in the chain rule schema being formed and coordinate them. (iii) At the Translevel a student has constructed the underlying structure of the chain rule. He or she links the composition and decomposition of functions to differentiation, and recognizes various forms of the chain rule as linked in the sense that they follow from the same general rule through function composition. It is only at this stage of development that the underlying structure of the chain rule schema is constructed through reflection on relationships between various objects from previous stages. At Trans- level the elements in the schema must go beyond being described essentially by a list, to being described by a single rule (Clark et al., 1997).

Based on the above, I arrived at the following genetic decomposition: For a student to have his or her function schema

(i) He will have developed a process or object conception of a function and 
(ii) Has developed a process or object conception of a composition of functions.

For a chain rule schema,

(iii) He or she will have developed a process conception of differentiation

(iv) The student will then use the previously constructed schemas of functions, composition of functions and derivative to define the chain rule. In this process the student has to recognize a given function as the composition of two functions, take their derivatives separately and the multiply them.

(v) The student recognizes and applies the chain rule to specific situations.

\section{Methodology}

The design and implementation of instruction in this study was guided by the proposed genetic decomposition together with Dubinsky' (1991) Activities, Class discussions and exercises (ACE), model. Activities were chosen and done in class with students so as to induce the relevant mental constructs associated with the chain rule schema. Activities designed to help students make the mental constructions in the proposed genetic decomposition were given to students in the form of a tutorial test. Students worked collaboratively and some individually. Through these activities the students gained experience with mathematical issues related to understanding the chain rule and helped students to gain experience in constructing actions corresponding to the concept.

Class discussions came after the students had received three consecutive lessons on the chain rule and its applications. Those lessons and instruction were video-taped and later video clips of discussions related to mental constructions of the chain rule and the thought process were recorded. Exercises were assigned to the students in the form of homework done outside of classroom supervision at the end of each of the consecutive lessons taught on chain rule definition and applications in calculus. The purpose of the exercises was for students to reinforce the ideas and concepts constructed in class and to use and apply the chain rule they had learnt. Exercises in the learning of the chain rule were also given in the form of written questions and answers in the form of tests, the specially designed questionnaire and in-depth interviews of students chosen according to answers given in the pen and paper exercises

Once a week, the class met in groups of $+/-30$ for tutorial lessons and on the other days in a regular classroom as a cluster of 197 for lessons. The purpose of the first lesson was to introduce the learners to the concept of the chain rule and how it could be used to differentiate composite trigonometric functions. It was important to know how an APOS analysis could help the researcher to understand the learning process by providing explanations on phenomena observed when students tried to construct understanding of the chain rule. In the second lesson, exercises were given to students to consolidate the understanding of the concept of the chain rule and to ascertain that all students in the group shared the same understanding of the concept and its applications. Exercises ranged in their level of difficulty from the simple one to more complicated differentiation problem. The third lesson involved integration by substitution using the chain rule. Various examples were demonstrated by the researcher with full explanations on the u-substitution and the use of all the techniques. The aim was to provide students with opportunities to make applications of the chain rule they learnt and prepare them for the mathematics in which chain rule would be applied.

Peer collaboration was encouraged by allowing students to work on mathematical tasks given during the lesson. This collaboration, when combined with effective sequencing and pacing of the lesson, contributes to the teaching and learning of mathematics. Pacing and sequencing refers to the way the lecturer moves from one concept to the next within the mathematics topic to ensure maximum use of instruction time. Feedback was also used by the lecturer to create an appropriate milieu for effective teaching and learning. There was greater lecturer-student interaction promoted as students were encouraged to verbalise what they saw and thought. It was through the students' comments, questions and answers that their mental constructions regarding the chain rule were accessed. This was done in an attempt to answer the question, 'How do students construct various structures to recognize and apply the chain rule in the context of calculus?'

\section{Discussions}

The approach of comparisons between three different techniques of differentiation of loaded trigonometric functions, were used to introduce the chain rule differentiation in lesson one. The first technique was the one using 'Leibniz form technique'. The second one was the one where we differentiate from the innermost function and move outwards called 'link form technique' of the chain rule. The third one involves straight application of the chain rule in differentiation and is known as a 'straight form technique'. In this technique students used the chain rule mechanically by finding the 
derivatives of all the functions starting with the function on the outside of the given problem and multiplying out. For example, consider differentiating $y=\ln \sin x^{3}$. Three forms of the chain rule have been characterised: (1) Leibniz form technique gives, we let $y=\ln u$; then $\frac{d y}{d u}=\frac{1}{u}$; where $u=\sin v$; and $\quad v=x^{3} \quad$ so that $\frac{d v}{d x}=3 x^{2} ;$ and $\frac{d u}{d v}=\cos u$, and $\frac{d y}{d x}=\frac{d y}{d u} \times \frac{d u}{d v} \times \frac{d v}{d x}=\frac{1}{u} \times \cos u \times 3 x^{2}$. This would give $\frac{3 x^{2} \cos x^{3}}{\sin x^{3}}$. (2) Link form technique gives, we get $3 x^{2} \times \cos x^{3} \times \frac{1}{\sin x^{3}}$. (3) Using the Straight form technique we get, $\frac{1}{\sin x^{3}} \times \cos x^{3} \times 3 x^{2}$. Answers using the three techniques were simplified to see if they were the same. Various examples on differentiation using the chain rule were discussed with the students in this class. These included differentiation of composite functions like: (1) $f(x)=\sin (\tan 3 x)$; (2) $y=\operatorname{cosec}(x+\cos x)$ (3) $y=\sin ^{3}(4 x)$ (4) $y=\ln \left(x^{2} \cdot \cos x\right)(5) f(x)=x^{2 x}$ and $(6)^{f(x)=\ln \frac{x^{5}\left(1-x^{2}\right)^{6}}{x\left(\sqrt{1-x^{3}}\right)}}$.

After the second problem, one of the students asked, 'Is it a must that all the time when differentiating we start at the end of the problem, can't we start from the beginning, will that make a difference?' The technique used to differentiate $y=\operatorname{cosec}(x+\cos x)$ was the straight form technique. Differentiation using the chain rule proceeded from the outward function to the innermost function. This was then presented as a solution, $y^{\prime}=-\cos e c(x+\cos x) \cot (x+\cos x) \cdot(1+(-\sin x))$.

A mental or physical transformation of mental or physical objects is considered to be an action when it is a reaction to stimuli which the subject perceives as external (Dubinsky, (1991). This student perceived and reflected on a repeated action of differentiation and wanted to establish control over it. This is why he wanted to verify the direction and steps to be followed when differentiating. The student operated in the action stage at that moment and was thinking about the problem in a step-by-step manner and was looking at one step at a time. The interiorisation of the action then started when the mental mechanism of differentiating composite functions was converted to a process that took place internally in the student's mind. Interiorisation had not been experienced by the said student at that point in time.

The students were working on their own on exercises given after which they exchanged books for corrections. Of most interest was the argument that ensued as each of the students in a pair argued for the correctness of his response and the other one also claiming his presentation to be correct. The dispute was around the different approaches that the two had followed in finding the solution to: Differentiate $y=\sin ^{3}(4 x)$. One of the students presented his solution as $4 \cdot \cos (4 x) \cdot 3 \sin ^{2}(4 x)$ while the other one gave $3 \sin ^{2}(4 x) \cdot \cos (4 x) \cdot 4$. They knew that the two solutions would give the same

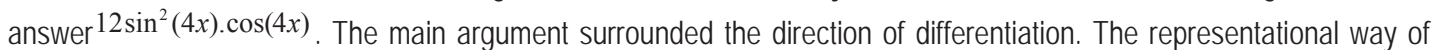
seeing the chain rule, was operational (seeing it from the inside) for one of them, while the other student saw it in a linear way, where he dealt with the differentiation one by one in a straight line. The latter student used the straight form technique of the chain rule while the former used the link form technique. Those students are said to be reacting to stimuli perceived as external in the action stage. They were treating differentiation as a formula. When they finally realised that both their solutions were correct, the action understanding was converted to process understanding where transformations were interiorised in their minds. Each one of them compared and learnt from each other the difference in approach to the same problem.

As the researcher went round from desk to desk checking on the answers displayed, she noticed how one student was arguing with his companion who marked him wrong for a presentation he thought was correct. The answer is displayed in Extract 1.

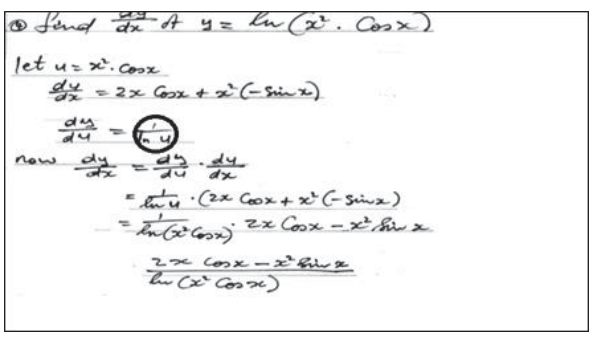

\section{Extract 1: S2's response}

The marker, S1, drew a circle and question mark in the problem above arguing that S2 (the owner of the book he marked) could not represent $\frac{d y}{d x}$ with a function in $u$ and $x$ at the same time. Initially he questioned even the method used 
by S2. When he was reminded of the Leibniz form technique used by Senzo who was comfortable with the substitution method, he (S1) exclaimed that 'It's a long method anyway.' S1 was operating in the action stage. He only knew how to perform operations on differentiation from memory or clearly given instruction. S2 had a process conception since he displayed transformation of physical or mental objects perceived as relatively internal and totally under his control. S2's error was to write the derivative of $y=\ln u$ as $\frac{d y}{d u}=\frac{1}{\ln u}$ instead of $\frac{1}{u}$. He was able to convince and justify by explaining to S1 how he found his derivative without deviating from the method used by S1 in his own solution. It was during redoing the problem and trying to convince $\mathrm{S} 1$ that he noted his mistake and rewrote the derivative as $\frac{1}{u}$. The argument was most interesting as S2 revisited his presentation to convince S1 that he understood the use of the Leibniz form technique and preferred it over the other techniques. S2 used conceptual understanding as he identified and applied differentiation principles. The students operating in the action stage care about getting the solution correctly and cannot justify how they arrived at their answers. They think about procedure in terms of its individual steps. They also could not link and adjust their steps of operations to differentiate functions comprising of multiple compositions. S2 had interiorized actions since he was able to use the process as an internalized procedure.

Subsequent activities were provided to help students build experiences of reconstructing familiar actions as general processes. Those activities were followed by higher order activities where the students needed to organize a variety of previously constructed objects, like functions and derivatives of composition of functions, into a schema that could be applied to problem situations involving the chain rule. More specifically their understanding of functions, composition of functions and the chain rule were evident from the students' attempts to answer the tasks given in class, their tests, and exercises.

Probing questions were used to elicit information on the chain rule more fully. This question type was used extensively in this study because the researcher wanted to explore students' thinking processes. Misconceptions and difficulties that emerged during the discussions were analysed with the view of establishing how students construct various structures to recognize and apply the chain rule in the context of calculus.

In lesson two, each student who had finished doing his/her problem was asked to read out his/her answer so that the researcher displayed it on the chalkboard. This was done to ensure that all the students in class got the correct solution to each problem with discussions and explanations of how one arrived at his/her answer. It was emphasized that in the solutions that were read out by students, it was not about a correct or wrong answer, but rather how he/she arrived at the particular answer. All the solutions to the given problems were displayed on the chalkboard. This helped even other students to check with their own solutions regarding each problem.

During this process, the emphasis was on: (1) discussions, (2) reflection explanations by the researcher where appropriate, (3) completion of the tasks by the students, and (4) understanding the use and application of the chain rule. It was so interesting to watch the students refering back to their notes in their books before attempting the questions. Asked about this S3 answered: I remember a problem that you did for us, it looked like this one. So I want to compare and then differentiate this one. Although S3 was operating in the action stage, he needed to gain experience constructing actions similar and corresponding to differentiating using the chain rule. The experience of differentiation using the chain rule was built upon in subsequent activities like those in the worksheet, where he was asked to reconstruct familiar actions as general manipulations.

One of the students applied the chain rule to the first task $y=\tan ^{2}\left(3 x+e^{\sqrt{x^{2}+1}}\right)$ correctly using the straight form technique. Some responses where the same technique was used, presented a solution with brackets, when they differentiated the composite function inside the brackets in the given task. One student left out the bracket then went on to detach the derivative 3 of $3 x$ from the + sign. This 3 now multiplied the first two functions (see Extract 2).

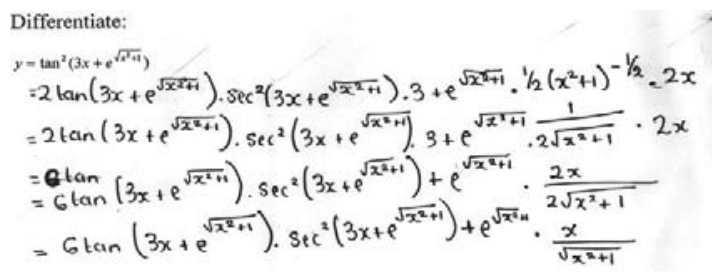

Extract 2: One student's presentation of a task 
Such students struggled with the connection of previously learnt algebraic skills like use of brackets where appropriate and manipulation of algebraic terms in a function. The calculations presented after differentiating using the chain rule successfully were therefore not correct. The mental images constructed by such students in using the chain rule were incomplete. Although the actions were interiorized into processes, the processes were not encapsulated to objects. This could partly be attributed to previous knowledge of algebraic skills which were just actions and never interiorized. According to the Triad students in the said students saw the chain rule as a procedure of differentiation which could not be connected or related to other processes applied to functions. Thus most students operated in the Intra- stage regarding the given task. According to APOS, we observed that some students could only go as far as the action stage.

The last task involved differentiating $y=\sqrt[3]{\frac{x(x+2)}{\left(x^{2}+1\right)}}$ by applying the chain rule.

Generally, one of two strategies was employed by students. The first form technique called for a specific connection between application of natural logarithms and differentiation.

Some students employed the straight form technique after converting the surd form to its exponential form. However, they did not then utilize the product and quotient rules appropriately. Their actions were not interiorized with regards to logarithms and this had an impact on applying the chain rule in the given task. Their mental images could not be related to the string of symbols forming the expression, since they could not interpret both the symbols and or manipulations. Since calculations reflect the active part of mental constructions, the rules for these students were not perceived as entities on which actions could be made. Dubinsky (2010) asserts that in such cases the difficulty does not depend on the nature of the formal expressions, but rather in the loss of the connections between the expressions and the situation instructions.

They would then work on it as individuals after which they compared their answers. Students argued and agreed upon certain responses. Individuals justified how they arrived at their responses. This way they taught each other and gave verbal descriptions of actions taken in their own words. They then repeated the actions many times with different tasks in their books and in the worksheet. Thus the worksheet helped the students interiorise the actions.

It was also noticed that most students were operating in the Intra- stage of the Triad. They had a collection of rules of differentiation with no recognition of relationships between them. Those students were helped by others who reflected on using the chain rule by applying actions to dynamic processes. The latter group of students had created an object of the chain rule. At the same time they applied actions on differentiation and as such the process of differentiating using the chain rule was encapsulated to form an object.

The responses to the problems in the worksheets were analyzed for meaning which is one of the mechanisms necessary for understsnding a concept. These included detecting (1) the connections made by students to other concepts, (2) calculations made using the chain rule, (3) the chain rule technique used, and (4) mental images on which the chain rule is based.

According to the Triad, students in the said groups saw the chain rule as a procedure of differentiation which could not be connected or related to other processes applied to functions. Thus most students operated in the Intra- stage regarding many tasks. This concurs with what Lakof \& Nunez (1997) who asserted that mathematics begins with direct human experience and ends there for some people.

The principles of effective mathematics teaching drawn from educational theories of Piaget illustrated that learning required interaction to develop: (1) a deep conceptual understanding, (2) positive relationships and (3) a classroom community. This social interaction leads to gradual, incremental changes in thought and behaviour of learners and through which interaction with other learners, allows them to examine, clarify and change their conceptual understanding. This study sought to explore how actions, processes and objects of the chain rule schema could be coordinated as mental structures to enhance the learning of the concept and access it in situations where it needs to be applied.

\section{Conclusion}

The students' responses discussed above indicate that the instructional pedagogy should accommodate presentation of tasks that evoke rigorous deductive reasoning enabling the students to write and reflect on how they construct various mental images. A wide range of interactions between students themselves and between students and the researcher were discussed. 


\section{References}

Anghileri, J. (2006). Scaffolding practices that enhance mathematics learning. Journal of Mathematics Teacher Education, $9,33-52$.

Asiala, M., Brown, A., Devries, D.J., Dubinsky E., Mathews, D., \& Thomas, K. (2004). A Framework for Research and Curriculum Development in Undergraduate Mathematics Education Research in Collegiate Mathematics Education II, Issues in Mathematics Education (CBMS), American Mathematical Society.

Berth, E. W., \& Piaget, J. (1966). Mathematical Epistemology and Psychology (W. Mays, trans.), Dodtrecht: Reidel.

Bransford, J., Brown, A., \& Cocking, R. (2000). How people learn: Brain, mind, experience, and school. Washington, DC: National Academy Press.

Brush, P.A., \& Saye, J. (2001). The use of embedded scaffolds with hypermedia-supported student-centered learning. Journal of Educational Multimedia and Hypermedia, 10 (1), 333 - 356.

Clark, J. M., Cordero, F., Cottrill, J., Czarnocha, B., DeVries, D. J., St. John, D., Tolias, T., \& Vidakovic, D. (1997). Constructing a schema: The case of the chain rule. Journal of Mathematical Behavior, 16, 345-364.

Confrey, J. (1994). Six Approaches to Transformation of Function Using Multi-Representational Software, Proceedings of the 18th Conference of the International Group for the Psychology of Mathematics Education, University of Lisbon, Portugal, Vol. 2, 217224.

Cottrill, J. (1999) Students' understanding of the concept of chain rule in first year calculus and the relation to their understanding of composition of functions, Ph.D. thesis, Purdue University.

Dubinsky, E. (1991). Reflective Abstraction in Advanced Mathematical Thinking. In David O. Tall (Ed.), Advanced Mathematical Thinking (pp. 95-123). Kluwer: Dordrecht.

Dubinsky, E., (1991a). 'Reflective Abstraction in advanced Mathematical Thinking', in: Tall, D., ed, Advanced Mathematical Thinking, The Netherlands: Kluwer.

Dubinsky, E. (2010). In Proceedings of the eighteenth annual meeting of the SAARMSTE: School of Science, Mathematics and Technology Education, University of KwaZulu Natal.

Dubinsky, E. and McDonald M.A. (2001), APOS: A constructivist theory of learning in Undergraduate Mathematics Education Research in the Teaching and learning of Mathematics at University level: An ICMI study. Kluwer Academic Publishers: Netherlands.

Ernest, P. (1991). The Philosophy of Mathematics Education. London: The Falmer Press.

Kaplan, W., (1984). 'Derivatives and differentials of composite functions' and 'the general chain rule' in advanced calculus. $3^{\text {rd }}$ Ed. Reading. MA: ADISON Wesley, pp101-105 and 106-110.

Gagnon, G., \& Collay, M. (2001). Designing for learning: Six elements in constructivist classrooms. Thousand Oaks, CA: Corwin Press.

Hapasaalo T. (2004). On instrumental genesis within Procedural and conceptual thinking. University of Joensuu, Finland.

Hiebert, J., \& Carpenter, T. P. (1992). Learning and teaching with understanding. In D. A.Grouws (Ed.), Handbook of research on mathematics teaching and learning (pp. 65-97). New York: Mcmillan.

Jojo, Z.M.M., Brijlall, D., \& Maharaj, A. (2011). The reliability of a research instrument used to measure mental constructs in the learning of the chain rule in calculus. Proceedings of the $16^{\text {th }}$ Annual Congress of the Association of Mathematics Education of South Africa (pp 336-349). Johannesburg: AMESA.

Jojo, Z.M.M. (2013) Mathematics begins with direct human experience. An APOS approach to conceptual understanding of a mathematical concept. ISTE Proceedings, UNISA Istitute for science and Technology

Jojo, Z.M.M., Brijlall, D. \& Maharaj, A. (2013). Schema development for the chain rule: A South African case. SAJHE Vol (27) 3.

Lutzer, C.V. (2003). Using motion to teach chain rule and u-substitution. PRIMUS, 13(1), 47-54.

Maharaj, A. (2010). An APOS Analysis of Student's Understanding of the Concept of a Limit of a Function. Pythagoras, 71, 41-51.

Mccosker, N. \& Diezman, C.,( 2009). Scaffolding students' thinking in mathematical investigations. APMC, 14 (3), 27 - 32.

Novotna, J., \& Hoch, M. (2008). How structure sense for algebraic expressions or equations is related to structure sense for abstract algebra. Mathematics Education Research Journal, 20(2), 93-104.

Piaget, J. (1966). Mathematical Epistemology and Psychology. Journal of Research in Science Teaching, 3, 301-326, Reidel.

Piaget, J. (1972). The Principles of Genetic Epistemology (W. Mays trans.) London: Routledge \& Kegan Paul.

Piaget, J. (1975). The Equilibration of cognitive structures. Cambridge: Harvard University Press.

Piaget, J. (1980). Adaptation and intelligence: Organic selection and Photocopy. Chicago: University of Chicago Press.

Piaget, J. (1985): The Equilibrium of Cognitive Structures. Harvard University Press, Cambridge, Massachusette: USA.

Piaget, J., \& Garcia, R. (1983). Psychogenèse et Histoire des Sciences. Paris: Flammarion.

Piaget, J., \& Garcia, R. (1989). Psychogenesis and the history of science (H. Feider, Trans.). New York: Columbia University Press. (Original work published 1983).

Pirie, S., \& Kieren, T. (1994). Growth in mathematical understanding: How can we characterize it and how can we represent it? Educational Studies in Mathematics, 26, 165-190.

Thoo, J. B. (1995). Composition and the chain rule using arrow diagrams. PRIMUS, 5(3), 291-295.

South Australian Certificate of Education [SACE] Board of South Australia. (2009). Curriculum statement 2009. Retrieved from http://www.ssabsa.sa.edu.au/docs/cs-2009/2mme-cs-2009.pdf.

Tall, D. O. (2007). Embodiment, symbolism and formalism in undergraduate mathematics education. Electronic proceedings of the 10th Conference of the Special Interest Group of the Mathematical Association of America on Research in Undergraduate Mathematics Education. San Diego, CA. Retrieved from http://sigmaa.maa.org/rume/crume2007/ eproc.html. 
Uygur, T., \& Ozdas, A. (2005) Misconceptions and difficulties with the chain rule. In The mathematics Education into the 21 st century Project, University of Teknologi Malaysia, Johor Bahru: Malaysia.

Uygur, T. (2010). Cognitive development of applying the chain rule through the three worlds. Australian Senior Mathematics JournalPublisher: The Australian Association of Mathematics Teachers, Inc. Audience: Academic. Vol. 24(2), pp 89-103.

Vygotsky, (1986). Thought and language. Cambridge, MA: Havard MIT Pres.

Weyer, S.R. (2010). APOS theory as a conceptualization for understanding mathematics learning. pp 9-15. Http://ripon.edu/macs/summation.

Wiggins, G., \& McTighe, J. (1998). Understanding by design. Alexandria, VA: Association of Supervision and Curriculum Development. 